\title{
The burden of TB-HIV in the EU: how much do we know? A survey of surveillance practices and results
}

\author{
M.E. Kruijshaar*, L. Pimpin*, I. Abubakar*, , B. Rice`, V. Delpech", L.N. Drumright*,+, \\ V. Hollo ${ }^{\S}$, E. Huitric ${ }^{\S}$, M. van de Laar $^{\S}$, A. Amato-Gauci ${ }^{\S}$, D. Manissero ${ }^{\S}$ and C. Ködmön ${ }^{\S}$
}

ABSTRACT: Information on the burden of tuberculosis (TB)-HIV co-infection is critical for the planning and evaluation of TB-HIV control and treatment strategies. This study assessed current practices in countries of the European Union (EU) and European Economic Area (EEA) for monitoring HIV co-infection in TB surveillance systems, countries' current co-infection burden and associated clinical practice.

An online survey was distributed to all national TB surveillance nominated European Centre for Disease Prevention and Control contact points in the EU/EEA.

We received 25 responses from 30 countries (83\% response rate). Patients' HIV status was collected in 18 out of the 25 TB surveillance systems, usually via clinician reporting (16 out of 18 surveillance systems). Although most countries recommended routine testing of TB patients for HIV, the proportion actually tested varied from $5 \%$ to $90 \%$. The burden of HIV co-infection was found to be elevated in countries with higher levels of HIV testing and higher prevalence of HIV.

We suggest that TB-HIV co-infection be monitored in all EU/EEA countries to facilitate the planning and evaluation of TB-HIV control strategies. Strengthening collaboration between TB and HIV clinicians and surveillance departments, and consideration of patient confidentiality restraints would be advantageous. The level of HIV testing in TB patients is low despite national recommendations and testing should be further promoted and monitored.

KEYWORDS: Co-infection, Europe, HIV/AIDS, surveillance, tuberculosis

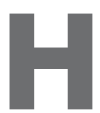

IV infection is an important risk factor for the development of active tuberculosis (TB) among individuals infected with $\mathrm{Myco-}$ bacterium tuberculosis and is associated with a higher case fatality amongst TB cases [1, 2]. Furthermore, the diagnoses of $M$. tuberculosis infection and active TB are complicated in people living with HIV as a result of immune suppression and atypical presentations [3, 4]. Treatment of patients with both conditions can be problematic due to drug interactions and overlapping toxicity profiles of the drugs involved [4]. The introduction of highly active antiretroviral therapy has been an important improvement, reducing the incidence of TB among people living with HIV and mortality of HIVpositive TB cases [5].

In Europe, recent trends in migration, and transmission of TB and HIV in hard-to-reach communities and congregate settings, may result in increasing numbers of TB-HIV co-infected cases [6-9]. The latest data for the European Union (EU) and European Economic Area (EEA; EU plus Iceland, Liechtenstein and Norway) show that between $0 \%$ and $15 \%$ of TB cases are co-infected with HIV in the $30 \mathrm{EU} / \mathrm{EEA}$ Member States with a varying degree of HIV testing coverage among TB patients [10].

All EU/EEA countries have reported case-based data to the European Centre for Disease Prevention and Control (ECDC) since 2008 and before this, reported either aggregated or case-based data to the EuroTB project (1996-2007) [10]. Information on the HIV status of TB patients is currently only reported to ECDC at aggregated levels by 16 countries.

To appropriately plan and evaluate TB-HIV control measures and treatment strategies, it is critical to have information on the burden of TB-HIV coinfection and monitor changes therein, particularly among population groups at risk. Herein, we present the results of a survey conducted in EU/EEA
AFFILIATIONS

*Tuberculosis Section, Respiratory Diseases Dept,

"HIV and AIDS Reporting Section, HIV and STI Dept, Health Protection Services Colindale, Health Protection Agency,

${ }^{+}$Centre for Infection Prevention and Management, Division of Infectious

Diseases and Immunity, Dept of

Medicine, Imperial College London, London,

${ }^{\#}$ School of Medicine, Health Policy and Practice, University of East

Anglia, Norwich, UK

${ }^{\S}$ European Centre for Disease Prevention and Control, Stockholm, Sweden.

CORRESPONDENCE

C. Ködmön

European Centre for Disease

Prevention and Control

Tomtebodavägen $11 \mathrm{~A}$

S-171 83 Stockholm

Sweden

E-mail: Csaba.Kodmon@

ecdc.europa.eu

Received:

Dec 222010

Accepted after revision:

May 042011

First published online:

June 302011 
countries on the current practices for monitoring HIV co-infection in TB surveillance systems, and provide suggestions for future surveillance and treatment.

\section{METHODS}

\section{Data collection and questionnaire development}

A web-based questionnaire was developed for data collection (Sawtooth Software, Sequim, WA, USA) and sent to national TB surveillance ECDC contact points in all EU/EEA countries.

Questionnaire development included several rounds of commenting by experts from the project group, including the UK national correspondents for TB and HIV surveillance and the ECDC TB and HIV surveillance leads. A final version was piloted amongst UK staff working in TB or HIV surveillance from different language backgrounds and countries of origin. The full questionnaire is available on request from the ECDC [11].

The questionnaire consisted of four parts (table 1). Questions focussed on two broad areas: 1) surveillance, including the collection of HIV status for reported TB cases (case-based data) and perceived limitations and support for TB-HIV co-infection surveillance; and 2) burden and clinical practice, including the estimated burden of HIV co-infection amongst TB patients in each country, distribution of the burden by patient characteristics, and national guidance and testing practices. There were, on average, 40 closed- and four open-response questions, depending on skip patterns. Closed-answer questions also contained "other" responses that were open-ended in order to capture information more accurately.

\section{Data quality}

The questionnaire included computer-automated checks for completeness and accuracy of data (e.g. numbers between 0 and 100 for a proportion). To verify the accuracy of the data and clarify any outstanding questions, all participants were sent the ECDC report for comments and corrections.

\section{Analysis}

Responses were collated and tabulated. To evaluate whether HIV co-infection levels amongst TB patients varied with the proportion of patients tested for HIV, the national HIV or TB burden, and/or population size, scatter plots were constructed and logarithmic trend lines fitted. National TB and HIV rates were obtained from the ECDC TB and HIV surveillance reports $[10,12]$. Population size was obtained from the EUROSTAT database (http:/ /ec.europa.eu/eurostat).

\section{RESULTS}

25 out of the $30 \mathrm{EU} / \mathrm{EEA}$ countries responded to the survey ( $83 \%$ response rate). Nonresponding countries had TB incidence and HIV prevalence levels in the same range as responding countries, but $60 \%$ never reported information on co-infection levels to ECDC (compared with $20 \%$ of responding countries).

Amongst responding countries, all TB surveillance systems had national coverage and reporting of TB was mandatory in all countries. Mobile populations, homeless people and migrants from countries with generalised TB and/or HIV epidemics were the groups most often thought to be under-reported in TB surveillance (eight, seven and five countries, respectively).

\section{Surveillance}

\section{Collection of HIV status}

In 18 (72\%) out of the 25 responding countries, HIV status was collected for individual TB patients reported to the national TB surveillance system. Information on the way co-infection status was collected in each country is provided in table 2 . In 16 $(89 \%)$ countries, the clinician provided HIV status on the TB case report, and in five countries, this was complemented by information from national HIV surveillance systems, HIV / AIDS laboratories or medical records; further details on methods were not collected. In two (11\%) countries, HIV coinfection status was obtained only through the matching of case records in the national TB and HIV surveillance databases.

HIV status is usually collected on a continuous basis. In one of the two countries that relied on matching, this was only performed every 2-3 yrs. The proportion of diagnosed HIV coinfected TB cases captured by the surveillance systems was

\section{TABLE 1 The four parts of the tuberculosis (TB)-HIV co-infection survey sent to European Centre for Disease Prevention and} Control TB contact points in European Union/European Economic Area countries

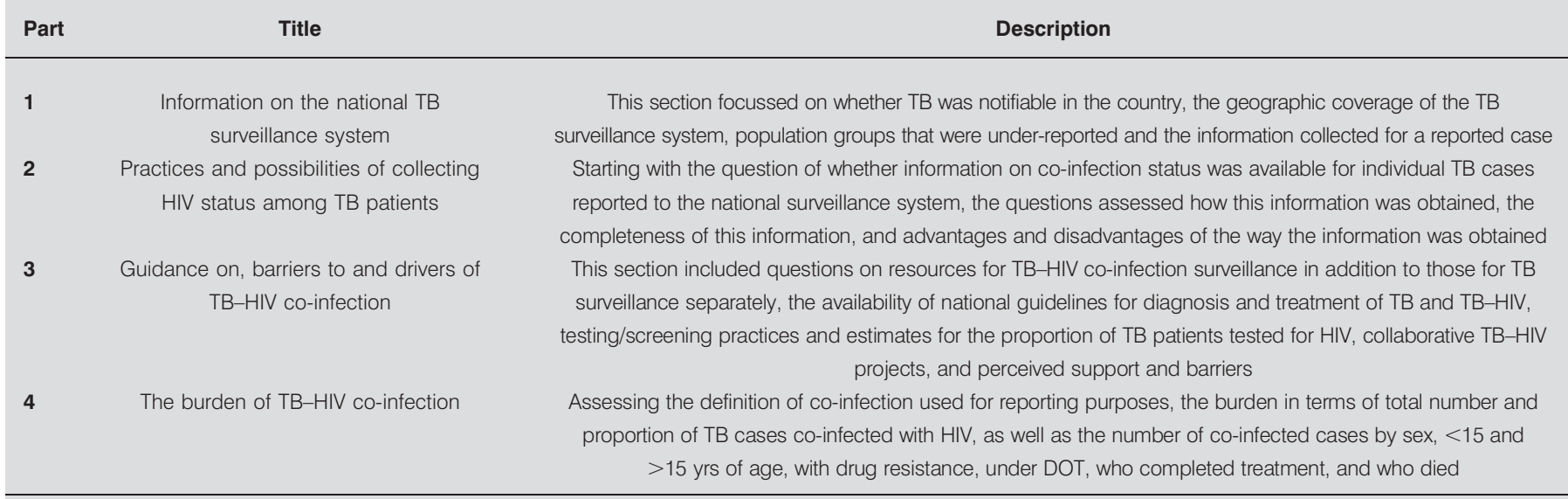

DOT: directly observed therapy 


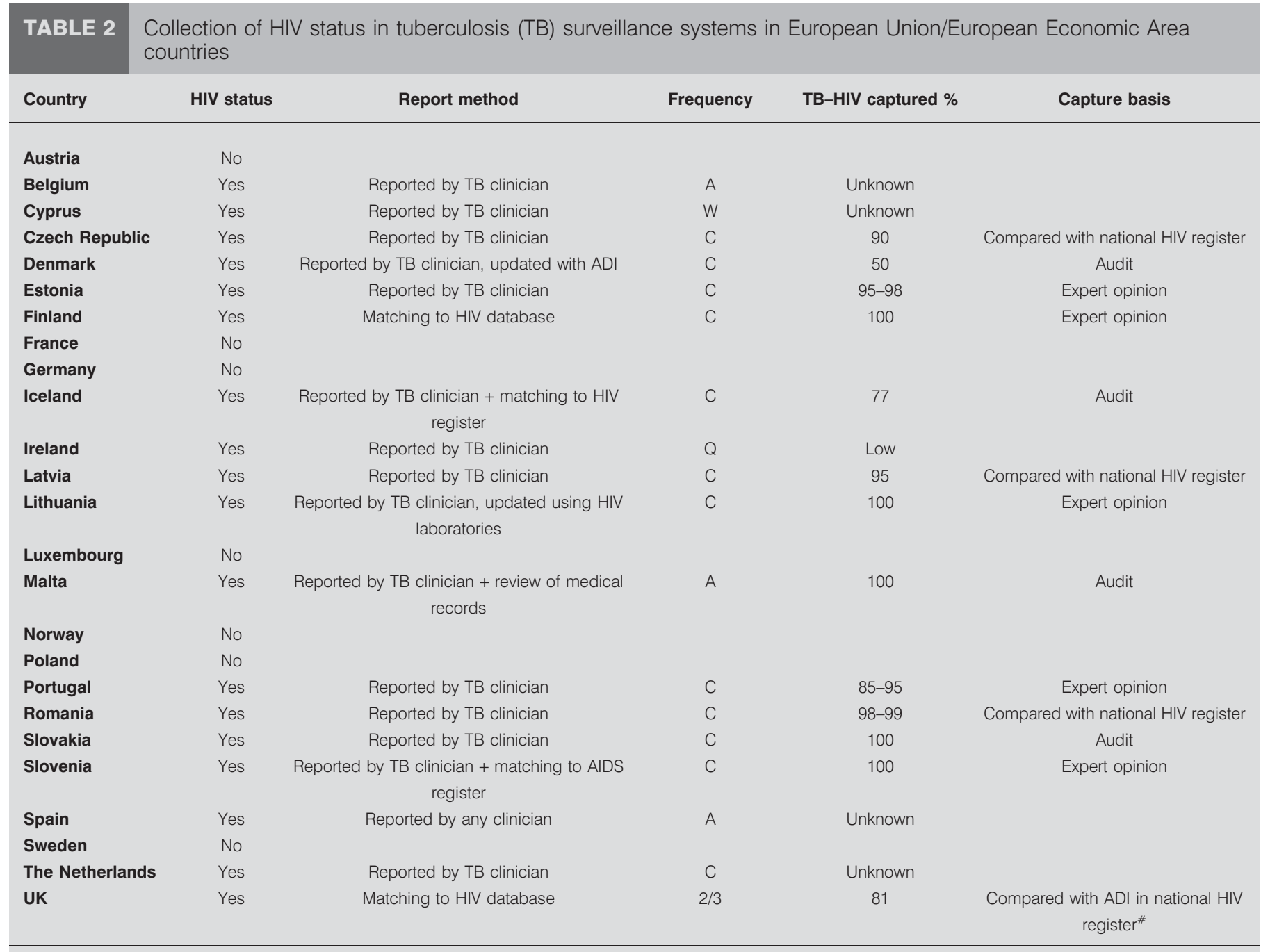

ADI: information on TB as AIDS-defining illness collected in the country; A: annually; W: when required; C: continuously; Q: quarterly; 2/3: reported every 2-3 yrs; Audit: comparison with patients' medical records. ${ }^{*}$ : cases not reported to TB register but included in HIV register with TB as AIDS-defining illness.

often not known; where provided, estimates varied from $50 \%$ to $100 \%$.

\section{Advantages of and barriers to TB-HIV co-infection surveillance}

Respondents from countries where HIV status was reported by clinicians in TB case reports highlighted that this has the advantages of posing little burden on clinicians (four (25\%) out of 16 respondents), being cheap (25\%) and requiring no special skills from the surveillance staff (31\%). It was seen as a weakness that not all HIV co-infected TB cases are captured by this method $(38 \%)$, mainly as a result of under-reporting by clinicians.

Updating HIV co-infection status by cross-checking or linking TB records with those pertaining to HIV/AIDS cases has the advantage of allowing the verification of co-infection status as reported to the TB and/or HIV surveillance system. Respondents from countries relying exclusively on matching believe this method ensures a high proportion of HIV co-infected TB cases are captured. The reported disadvantage is that it is time consuming and requires good coordination between those responsible for TB and HIV surveillance.

Representatives from countries that do not record HIV status for individual TB patients reported being restricted from collecting such information due to anonymous reporting of HIV cases and/or patient confidentiality legislation. These same two reasons were most frequently identified by all respondents as a barrier to TB-HIV co-infection surveillance (12 (48\%) out of 25 respondents). Poor collaboration between TB and HIV surveillance departments and clinicians (32\%), and lack of resources (20\%) were also mentioned.

\section{Burden and clinical practice}

Testing practices and guidelines

Nearly all responding countries had national policies recommending the testing of TB patients for HIV infection (22 $(88 \%)$ out of 25 countries) (table 3). Estimates of the proportion of cases actually tested varied widely from around 5\% to $90 \%$ and 12 


\section{TABLE 3 Policies for HIV testing in tuberculosis (TB) patients and programmes for targeted TB testing in risk groups in European} Union (EU)/European Economic Area countries

Country

Routine HIV testing of TB patients

\begin{tabular}{cc}
\hline Recommended & $\begin{array}{c}\text { Tested \% } \\
\text { (year, source) }\end{array}$
\end{tabular}

(year, source)
Risk groups with programmes for targeted TB testing

\begin{tabular}{|c|c|c|c|}
\hline Austria & Yes & Unknown & Homeless, IDUs, prisoners, migrants (any) \\
\hline Belgium & Yes & $72(2001-2008, S)$ & Homeless, IDUs, prisoners, asylum seekers \\
\hline Cyprus & Yes & Unknown & Prisoners, migrants (non-EU) \\
\hline Czech Republic & No & $20(2008, S)$ & Homeless, prisoners, migrants (gen) \\
\hline Estonia & Yes & $90(2008, \mathrm{~S})$ & Homeless, IDUs, prisoners, migrants (any), medical and school personnel \\
\hline Finland & No & Unknown & Refugees, asylum seekers \\
\hline Iceland & Yes & $77(2000-2009$, S) & Migrants (gen) \\
\hline Ireland & Yes & Unknown & Prisoners, asylum seekers \\
\hline Latvia & Yes & Unknown & Homeless, IDUs, prisoners, migrants (any) \\
\hline Lithuania & Yes $^{\#}$ & Unknown & None \\
\hline Luxembourg & Yes & Unknown & Prisoners, migrants (any) \\
\hline Malta & Yes & $85(2008, S)$ & Prisoners, migrants (gen) \\
\hline Slovakia & Yes & $90(2009$ A) & Homeless, prisoners, mobile populations, migrants (Roma) \\
\hline Slovenia & Yes & 50 (2007, C-R) & Homeless, IDUs, prisoners, migrants (any) \\
\hline Spain & Yes & $50(2008, S)$ & Homeless, IDUs, prisoners, migrants (gen) \\
\hline Sweden & Yes & Unknown & None \\
\hline The Netherlands & Yes & $26(2008, S)$ & Homeless, IDUs, prisoners, migrants (gen) \\
\hline UK & No & Unknown & Homeless, IDUs, prisoners, migrants (gen) \\
\hline
\end{tabular}

S: surveillance; A: audit; C-R: capture-recapture study; IDU: injecting drug user; gen: from countries with generalised TB and/or HIV epidemics. ${ }^{\#}$ : offered to all TB patients $>16$ yrs of age.

countries could not provide an estimate for this. National guidelines for the diagnosis and treatment of TB-HIV co-infected patients were available in $64 \%$ of the countries.

Programmes for targeted TB testing among migrant groups existed in most countries (92\%). Programmes for testing prisoners were also common $(76 \%)$, while about half the countries tested homeless persons $(60 \%)$. Testing programmes for injecting drug users were less common $(40 \%)$.

\section{Estimated burden of TB-HIV co-infection}

The number and proportion of reported TB patients co-infected with HIV are presented for each country in table 4 . The level of HIV co-infection varied between $0 \%$ and $15 \%$, with the highest proportions reported from Estonia, Portugal and Iceland. In general, countries in central Europe reported very low levels of TB-HIV co-infected patients.

France and Poland did not collect HIV status in individual TB case reports and the figures provided are based on aggregated information on AIDS cases reported with TB as an AIDS-defining illness.
Demographic and clinical characteristics of HIV co-infected TB cases

Of the 20 countries with available data on HIV co-infection among TB patients (the 18 countries collecting these data plus France and Poland), most could provide information on sex (16 $(80 \%)$ out of 20$), 70 \%$ on age, $65 \%$ on multidrug resistance (MDR) and deaths, and $45 \%$ on directly observed therapy (DOT) (table 4). There was a predominance of male patients in most countries, but in Belgium and Finland the sex ratio was equal, and in Iceland and Ireland the majority of patients were female. The high levels of MDR observed amongst TB-HIV co-infected patients in Lithuania, the Czech Republic and Estonia (10\%, 14\% and $20 \%$, respectively) should be interpreted with caution due to the small numbers of co-infected cases in these countries. The use of DOT varied widely between countries, with some reporting all TB-HIV co-infected patients receiving DOT and others none. Between $0 \%$ and $29 \%$ of co-infected patients died.

\section{Analysis of variation in TB-HIV burden}

The burden of HIV co-infection amongst TB patients was generally higher in countries with high levels of HIV testing among TB 


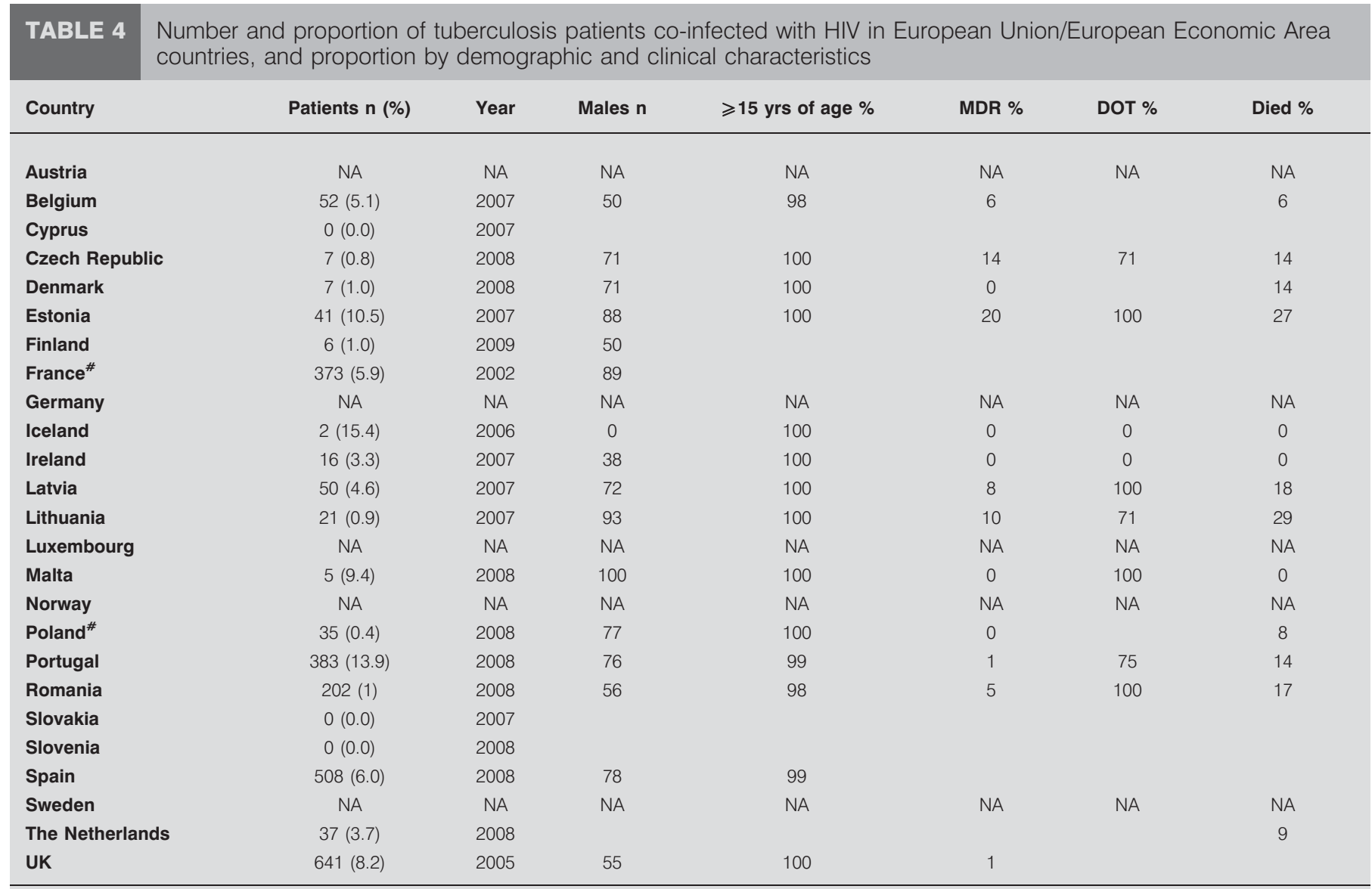

MDR: multidrug resistance; DOT: directly observed treatment; NA: not available because HIV status is not collected in the TB surveillance system. ${ }^{*}$ : HIV status is not collected in the TB surveillance system (figures are estimates based on the number of AIDS cases reported with TB as an AIDS-defining illness).

patients $(>70 \%)$ compared with those with lower levels of testing $(\leqslant 50 \%$ ) (fig. 1). Co-infection levels also tended to be higher in countries reporting a higher HIV prevalence [12]. There was no systematic variation by TB burden or population size of countries.

\section{DISCUSSION}

Our survey demonstrated that in the majority of EU/EEA countries, information on HIV status is collected for individual
TB patients reported to the national TB surveillance systems. However, the percentage of diagnosed TB-HIV cases reported to the surveillance systems remains suboptimal and varies throughout the EU/EEA. The main barriers to TB-HIV coinfection surveillance mentioned were patient confidentiality and the anonymous testing and reporting of HIV cases. Nearly all countries recommend testing all TB patients for HIV, although testing levels varied widely. The proportion of TB
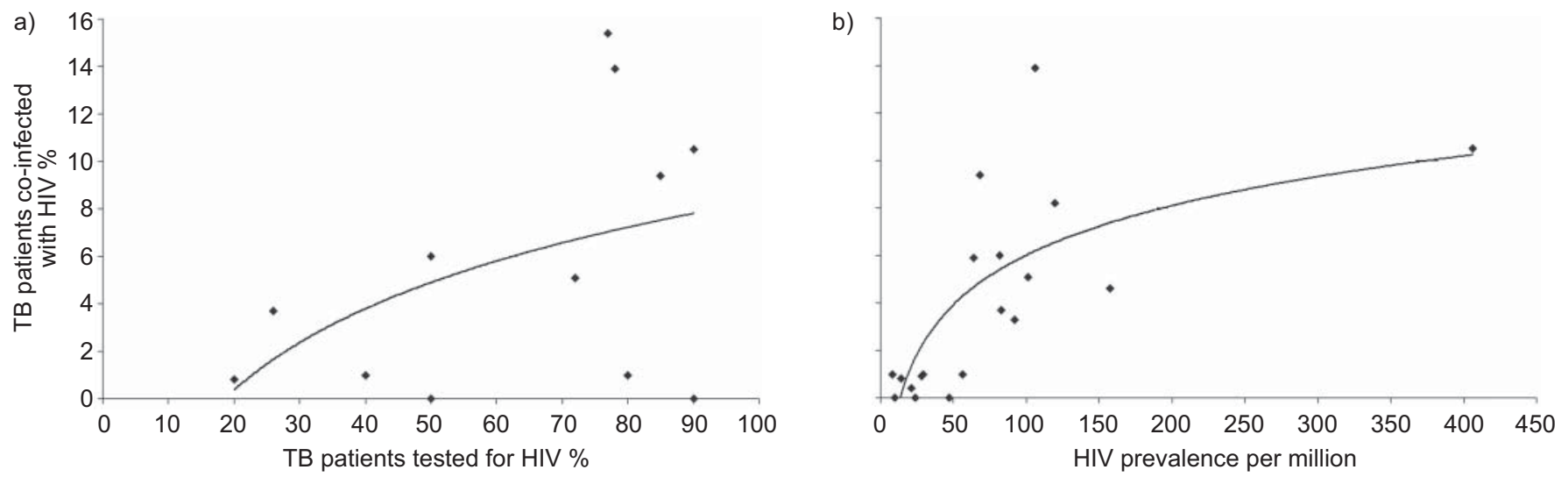

FIGURE 1. Proportion of tuberculosis (TB) patients co-infected with HIV by a) proportion of TB patients tested for HIV and b) national HIV/AIDS prevalence [12]. 
patients co-infected with HIV was influenced by the proportion of TB patients tested for HIV and the prevalence of HIV/ AIDS in the country.

\section{Strengths and limitations}

This survey had a high response rate, and TB incidence and HIV prevalence levels in the five nonresponding countries were in the same range as those of responding countries. Nevertheless, three out of these five countries had never reported information on the level of HIV co-infection amongst TB patients to ECDC, suggesting that the unavailability of coinfection data may have affected the response rate.

Many variables collected depended on subjective assessment of data by one national expert, potentially affecting the quality of data collected. Nevertheless, this survey has identified a number of areas and opportunities to improve the surveillance of TB-HIV co-infection both at the national and EU/EEA level.

Because of the limited number of data points, it is difficult to assess how well the trend lines represent the data points and one outlier may have very strong effects. Nevertheless, the general trend for higher levels of HIV co-infection amongst TB patients in settings with higher proportions of TB patients tested for HIV, as well as HIV prevalence, remains present after removing possible outliers.

\section{Collection of HIV status in TB surveillance}

The collection of information on HIV co-infection among TB patients is important to allow better planning of services and control programmes both at national and international level, as recommended by the World Health Organization (WHO) [13]. In contrast to an earlier study that reported countries often did not have a national surveillance system to monitor HIV among TB patients [14], our study shows that a large proportion of countries $(72 \%)$ appear to have such a system in place. The earlier study was conducted in selected countries of the WHO European Region and included only 11 out of the 30 EU/EEA countries. Although this difference in coverage may partly explain discrepancies, it is also possible that some EU/EEA countries have recently started collecting HIV co-infection status of TB cases.

HIV co-infection status was most commonly collected in the TB case report form. These data are reliant on the willingness of clinicians to report, which some national contacts indicated could be an issue, as well as their awareness of the patient's HIV status. The use of additional information from national HIV / AIDS surveillance, laboratories or medical records to update the available information would improve completeness of information and allow countries to estimate levels of underreporting, which was unknown in most countries. Whether such methods can be used will depend, however, on patient confidentiality requirements, staff skills and resources.

Matching national TB and HIV databases using automated matching algorithms was the sole data source in two countries. Although this method ensures that a high proportion of HIV coinfected TB patients are captured, estimates will depend on the completeness of reporting of TB and HIV cases separately. Drawbacks of matching include the inability to distinguish between patients with unknown HIV status and those with negative HIV status, and a lengthy matching process that reduces the timeliness with which the data become available.

Some countries that did not collect HIV co-infection status for individual TB patients did provide estimates of the burden of co-infection using AIDS case reports that listed TB as the AIDS-defining illness. Although it is preferential to have some estimate rather than none [15], it should be acknowledged that these are likely to underestimate the true proportion of TB patients co-infected with HIV, because subsequent diagnoses of TB after the first AIDS report are often not recorded in HIV/ AIDS surveillance systems [11].

Countries that did not collect information on TB-HIV coinfection pointed out that this was related to patient confidentiality and anonymous reporting issues. This is in agreement with the previous study by LAZARUS et al. [14]. Such regulations can be challenged and changed, as shown by one country reporting that the matching of $\mathrm{TB}$ and HIV data will be possible in the future as a result of a change in legislation. Anonymous surveys of HIV prevalence amongst TB cases can also be used if legislation does not allow the collection of HIV co-infection status in TB surveillance. These are, however, expensive, time consuming and only provide a snap shot (point prevalence).

In countries where HIV co-infection status of TB patients is collected, surveillance may be further supported by stimulating collaboration between TB and HIV surveillance departments and/or clinicians (which could also improve treatment coordination) and the consideration of national funding. Patient confidentiality should be ensured, for example, by storing identifiable information in an unlinked dataset. Anonymous case reporting is not preferential, as this would prevent matching or crosschecking with other databases, as well as de-duplication and denotification of cases.

\section{Burden of HIV co-infection amongst TB patients}

The highest proportions of TB patients co-infected with HIV were reported from Estonia, Portugal and Iceland, with very low levels in countries in central Europe, which is also confirmed in ECDC surveillance reports [10]. This pattern is in agreement with the high prevalence of HIV in Estonia and Portugal and low HIV prevalence rates in countries in central Europe [12]. Estimates were similar to those reported to ECDC [10], although small differences were seen as a result of further updating of surveillance data and new data being released.

In order to interpret and compare co-infection levels, information on the completeness of reporting and the proportion of cases tested for both diseases are crucial, and countries should aim to estimate these. Estimates of the proportion of HIV coinfected TB cases captured by surveillance systems varied widely in this survey and were not available for several countries. Under-reporting of TB cases may also play a role, e.g. groups at high risk of TB-HIV co-infection, such as migrants from countries with high prevalence for both diseases and injecting drug users, were identified as under-reported to national TB surveillance systems in several countries.

Our analysis showed that reported levels of HIV co-infection among TB patients were higher in countries with higher levels 
of HIV testing in this patient group. This could suggest that there is a larger proportion of undiagnosed HIV in countries with lower levels of testing. However, countries reporting more HIV testing in TB patients also had a higher prevalence of HIV [12], which was also shown to be associated with higher proportions of HIV co-infection. It is thus also possible that HIV prevalence drives the higher level of HIV co-infection and is the reason for testing $\mathrm{TB}$ patients more often in these countries.

\section{Testing practices and guidance}

WHO advocates universal testing of TB patients for HIV in populations with a concentrated HIV epidemic, which would include subpopulations such as injecting drug users in most EU/EEA countries [16]. Despite the fact that most countries (88\%) recommended routine HIV testing in TB patients, the proportion of cases actually tested was very low. This is in line with studies that have shown routine testing is not offered universally in countries where this is recommended, suggesting that there are missed opportunities for diagnosing HIV in TB patients [17-19]. Knowledge of HIV co-infection status is paramount to ensure proper treatment and care. Testing and screening practices should be further expanded and promoted in line with WHO recommendations.

Information on the proportion of cases tested is important for monitoring adherence to testing recommendations as well as to interpreting and comparing co-infection levels over time and between countries. About half the countries could estimate the proportion of TB patients tested for HIV.

National guidelines specifically for TB-HIV diagnosis and treatment were not available in a third of countries. This may be a result of limited resources and European guidance could be a useful resource for these countries.

\section{Conclusion}

Information on HIV status of TB patients was collected in TB surveillance systems in 18 out of 25 responding EU/EEA countries. Further efforts should be made to collect this information in all EU/EEA countries, and to improve and measure the completeness where it is already collected. Improving the collaboration between TB and HIV clinicians and surveillance departments, while working within the necessary data security framework, would be advantageous.

The level of HIV testing in TB patients is low in some countries despite national recommendations for routine testing. Testing for HIV should be further promoted and testing levels require monitoring in all countries. This is of crucial importance, particularly in EU/EEA settings, as antiretroviral treatment is relatively accessible in most EU/EEA countries.

In summary, despite the modest TB-HIV co-epidemic in the EU/EEA compared with high TB and HIV burden countries (e.g. Southern Africa), co-infection remains an important determinant of TB morbidity and mortality in the EU/EEA. Efforts should be made to optimise surveillance and maximise the effectiveness of control programmes among the TB population, particularly within vulnerable and at-risk populations.

\section{SUPPORT STATEMENT}

This work was funded by the European Centre for Disease Prevention and Control (service contract number ECD 1632).

\section{STATEMENT OF INTEREST}

None declared.

\section{ACKNOWLEDGEMENTS}

We thank all national contact points for TB surveillance in EU/EEA countries who participated in the survey for their contribution, and acknowledge G. Likatavicius and I. Devaux (both European Centre for Disease Prevention and Control, Stockholm, Sweden) for their input.

\section{REFERENCES}

1 Corbett EL, Watt CJ, Walker N, et al. The growing burden of tuberculosis: global trends and interactions with the HIV epidemic. Arch Intern Med 2003; 163: 1009-1021.

2 Lienhardt C, Rodrigues LC. Estimation of the impact of the human immunodeficiency virus infection on tuberculosis: tuberculosis risks re-visited? Int J Tuberc Lung Dis 1997; 1: 196-204.

3 Geng E, Kreiswirth B, Burzynski J, et al. Clinical and radiographic correlates of primary and reactivation tuberculosis: a molecular epidemiology study. JAMA 2005; 293: 2740-2745.

4 Sterling TR, Pham PA, Chaisson RE. HIV infection-related tuberculosis: clinical manifestations and treatment. Clin Infect Dis 2010; 50: Suppl. 3, S223-S230.

5 Haar CH, Cobelens FG, Kalisvaart NA, et al. HIV-related mortality among tuberculosis patients in The Netherlands, 1993-2001. Int J Tuberc Lung Dis 2007; 11: 1038-1041.

6 Ahmed AB, Abubakar I, Delpech V, et al. The growing impact of HIV infection on the epidemiology of tuberculosis in England and Wales: 1999-2003. Thorax 2007; 62: 672-676.

7 Hayward AC, Darton T, Van Tam JN, et al. Epidemiology and control of tuberculosis in Western European cities. Int $J$ Tuberc Lung Dis 2003; 7: 751-757.

8 Martin V, Cayla JA, Bolea A, et al. Mycobacterium tuberculosis and human immunodeficiency virus co-infection in intravenous drug users on admission to prison. Int J Tuberc Lung Dis 2000; 4: 41-46.

9 Velasco M, Castilla V, Cervero M, et al. The changing pattern of tuberculosis and HIV co-infection in immigrants and Spaniards in the last 20 years. HIV Med 2008; 9: 227-233.

10 European Centre for Disease Prevention and Control, WHO Regional Office for Europe. Tuberculosis Surveillance in Europe 2008. Stockholm, European Centre for Disease Prevention and Control, 2010.

11 European Centre for Disease Prevention and Control, WHO Regional Office for Europe. Survey on Tuberculosis and HIV Coinfection Surveillance in EU and EEA Countries 2009/2010. Stockholm, European Centre for Disease Prevention and Control, 2011.

12 European Centre for Disease Prevention and Control, WHO Regional Office for Europe. HIV/AIDS Surveillance in Europe 2008. Stockholm, European Centre for Disease Prevention and Control, 2010.

13 Stop TB Dept, Dept of HIV/AIDS, WHO, EFPAR and UNAIDS. A Guide to Monitoring and Evaluation for Collaborative TB/HIV Activities - 2009 revision. Geneva, World Health Organization, 2009.

14 Lazarus JV, Olsen M, Ditiu L, et al. Tuberculosis-HIV co-infection: policy and epidemiology in 25 countries in the WHO European region. HIV Med 2008; 9: 406-414.

15 Murray CJ, Lopez AD. Quantifying disability: data, methods and results. Bull World Health Organ 1994; 72: 481-494. 
16 TB/HIV working group of the Stop TB Partnership, WHO/ UNAIDS Working Group on Global HIV/AIDS/STI Surveillance. Guidelines for HIV Surveillance Among Tuberculosis Patients (2nd Edn). Geneva, World Health Organization, 2004.

17 Haar CH, Cobelens FGJ, Kalisvaart NA, et al. HIV-test policy for tuberculosis patients in the Netherlands. Ned Tijdschr Geneeskd 2007; 151: 2674-2679.
18 Rodger AJ, Story A, Fox Z, et al. HIV prevalence and testing practices among tuberculosis cases in London: a missed opportunity for HIV diagnosis? Thorax 2010; 65: 63-69.

19 Van der Werf MJ, Yegorova OB, Chechulin Y, et al. HIV testing practices of TB patients after introduction of a new testing policy in Kiev City, Ukraine. Int J Tuberc Lung Dis 2005; 9: 733-739. 